\title{
OPG, OPN, EGF and VEGF Levels at Individual Breslow Score Stages in Malignant Melanoma
}

\author{
INKA TRESKOVA ${ }^{1}$, ONDREJ TOPOLCAN ${ }^{2}$, JINDRA WINDRICHOVA ${ }^{2}$, \\ VACLAV SIMANEK ${ }^{2}$, DAVID SLOUKA ${ }^{2}$, VLADISLAV TRESKA $^{3}$ and RADEK KUCERA ${ }^{2}$ \\ ${ }^{1}$ Department of Plastic Surgery, University Hospital and Faculty of Medicine in Pilsen, Pilsen, Czech Republic; \\ ${ }^{2}$ Department of Immunochemistry, University Hospital and Faculty of Medicine in Pilsen, Pilsen, Czech Republic; \\ ${ }^{3}$ Department of Surgery, University Hospital and Faculty of Medicine in Pilsen, Pilsen, Czech Republic
}

\begin{abstract}
Background/Aim: Melanoma represents one of the most aggressive forms of cancer. With the rapid increases in the incidence of melanoma in the United States, Australia and Europe over the last decades, melanoma has been considered an epidemic cancer in these areas. The aim of our study was to evaluate the utility of osteoprotegerin $(O P G)$, osteopontin $(O P N)$, epidermal growth factor (EGF) and vascular endothelial growth factor VEGF for the diagnosis and prognosis of melanoma. Patients and Methods: Overall, 322 individuals were assessed: 183 melanoma patients and 139 healthy individuals. Melanoma patients were divided into four subgroups according to the Breslow score. OPN, OPG, EGF, and VEGF were determined in each plasma sample. Results: The serum levels of the following biomarkers were statistically significantly higher in the melanoma group compared to the control group: $O P G$ and, OPN $(p<0.0001), E G F(p=0.0379)$. In the first stage, $O P G(p=0.0236)$ and $O P N(p=0.0327)$ showed $a$ statistically significant increase. Concerning positive and negative sentinel node metastases a statistically significant change was observed in: OPN ( $p<0.0001)$, EGF $(p=0.0114)$, VEGF ( $p=0.0114)$. Conclusion: $O P G$ and $O P N$ are promising biomarkers of early-stage melanoma. EGF and VEGF appear to be prognostic biomarkers.
\end{abstract}

Melanoma represents one of the most aggressive forms of cancer. With the rapid increases in the incidence of melanoma in the United States, Australia and Europe over the last decades, melanoma has been considered an epidemic

Correspondence to: Asst. Prof. Radek Kucera, Department of Immunochemistry, University Hospital Pilsen, Edvarda Benese 13, 30599 Pilsen, Czech Republic. Tel: +420 377402948, Fax: +420 377402454, e-mail: kucerar@fnplzen.cz

Key Words: Melanoma, OPG, OPN, EGF, VEGF, Breslow score, sentinel node. cancer in these areas (1-3). The risk of melanoma increases with age and is gender-dependent. The average age at the time of diagnosis is about 60 years. Melanoma is approximately 1.5 times more frequent in males than in females (4). Nowadays, modern personalized medicine tends to use individual biomarkers to subdivide traditional tumour stages into subunits according to their different behavior. As melanoma is supposed to be a heterogeneous group of disorders, there is a need for individualization of melanoma diagnosis, prognosis and treatment. Melanoma biomarker research is an open field for research and understanding of molecular events in melanoma progression and should provide new molecular targets for therapeutic intervention $(5,6)$. The aim of our study was to evaluate the utility of four biomarkers: osteoprotegerin (OPG), osteopontin (OPN), epidermal growth factor (EGF) and vascular endothelial growth factor (VEGF) for the early diagnosis and prognosis of melanoma.

\section{Patients and Methods}

Group of patients. The total number of probands was 322. Two groups of probands were assessed: 183 patients with melanomas and 139 healthy persons. All melanoma diagnoses were histologically verified. The basic characteristics of melanoma and control groups is shown in Table I. The following biomarkers were determined in both groups: OPG, OPN, EGF and VEGF. The next step was to stratify melanoma patients into four groups according to melanoma thickness (Breslow score). biomarker levels were compared in these four groups with the group of healthy persons. Finally, the group of patients was assessed with metastatic sentinel node positivity and the group without metastases.

Plasma samples. Peripheral venous blood was collected using the VACUETTE blood collection system (Greiner Bio-one Company, Kremsmünster, Austria). Plasma was separated by $10 \mathrm{~min}$ of centrifugation at $1300 \mathrm{~g}$, and immediately frozen to $-80^{\circ} \mathrm{C}$. Plasma samples were collected at the time of diagnosis, prior to surgery or any other form of treatment. Samples were thawed only once, just prior to analyses. 
Methods used. Plasma levels of OPG and OPN were assayed using a Human bone panel 1 Milliplex Map kit (Millipore Corporation, Billerica, MA, USA). EGF and VEGF were assayed using a Human Cytokine/Chemokine Milliplex Map kit (Millipore Corporation, Billerica, MA, USA). Measurement was performed using the BioPlex MAGPIX Multiplex Reader (Bio-Rad Laboratories, Hercules, CA, USA).

Statistical methods. The SAS 9.2 (Statistical Analysis Software release 9.2; SAS Institute Inc., Cary, NC, USA) was used for all statistical analyses. A summary of statistical findings for age and biomarker levels is presented. The Wilcoxon test was used to compare distributions of values between the groups of patients at each Breslow score stage with the group of healthy probands. The Wilcoxon test was also used for the comparison of the group with metastatic sentinel node positivity and the group without metastatic positivity.

\section{Results}

An overview of the individual biomarker comparisons between the healthy and melanoma groups is shown in Table II. In the melanoma group, the results were higher with statistical significance for the following biomarkers: OPG (median=355.2, $p<0.0001$ ) compared to $234.1 \mathrm{pg} / \mathrm{ml}$ in the group of healthy probands, OPN (median $=14,957 \mathrm{pg} / \mathrm{ml}, p<0.0001$ ) compared to $8535 \mathrm{pg} / \mathrm{ml}$ and EGF (median=22.00 pg/ml, $p=0.0379$ ) compared to $29.00 \mathrm{pg} / \mathrm{ml}$. The change in VEGF levels was not significant. A detailed evaluation of biomarkers at Breslow score stages is shown in Table III. Biomarker levels in the group of healthy persons were compared with the biomarker levels at each Breslow score stage. We focused on the first Breslow score stage and its comparison with the group of healthy probands. A statistically significant difference was only observed in the levels of two biomarkers: OPG (median=287.6, $p=0.0239$ ) compared to $234.1 \mathrm{pg} / \mathrm{ml}$ in the group of healthy probands and OPN (median=12,801 pg/ml, $p=0.0327$ ) compared to 8535 $\mathrm{pg} / \mathrm{ml}$. Comparing the group of positive and negative sentinel node metastases a statistically significant change was observed in three biomarker levels: OPN (median of the negative group $=14247 \mathrm{pg} / \mathrm{ml}$, median of the positive group= $25,244 \mathrm{pg} / \mathrm{ml}, p<0.0021$ ), EGF (median of the negative group $=26.25 \mathrm{pg} / \mathrm{ml}$, median of the positive group $=80.15 \mathrm{pg} / \mathrm{ml}$, $p=0.0114$ ), VEGF (median of the negative group $=105.8 \mathrm{pg} / \mathrm{ml}$, median of the positive group=979.8 $\mathrm{pg} / \mathrm{ml}, p=0.0027$ ). We also calculated and compared the Breslow score of the negative and positive sentinel node group (median of the negative group= $1.8 \mathrm{~mm}$, median of the positive group $=2.9 \mathrm{~mm}, p=0.0004$ ). These results are shown in Table IV.

\section{Discussion}

Cutaneous melanoma is a highly malignant tumour. Treatment of early stage melanoma has the potential to be more successful than the treatment of melanoma at advanced stages.
Table I. Basic characteristics of melanoma and control group.

\begin{tabular}{llcc}
\hline Item description & & Melanoma group & Control group \\
\hline Female & $\mathrm{N} / \%$ & $102 / 56$ & $75 / 54$ \\
Male & $\mathrm{N} / \%$ & $81 / 44$ & $64 / 46$ \\
F+M total count & $\mathrm{N}$ & 183 & 139 \\
Age & Median (years) & 56.7 & 56.0 \\
& $($ min-max) & $(16-87)$ & $(17-84)$ \\
\hline
\end{tabular}

The Breslow thickness of the tumour (Breslow score) and the status of the sentinel lymph node are still the most important prognostic factors for disease free interval (DFI) and overall survival (OS). As primary tumour thickness increases, there is a significant decrease in survival (7). Patients with thin melanoma and negative sentinel lymph node can be assured that their risk of recurrence is extremely low (8). For early detection ability this study focused on the group with a Breslow score of less than $1 \mathrm{~mm}$ (Table III).

Afterwards the study was focused on biomarkers as prognostic factors. Current prognostic factors do not give us accurate information to predict melanoma behavior in individual patients, the aggressiveness of the disease, or the way a tumour might disseminate. The levels of four biomarkers were correlated with the sentinel lymph node status. The Breslow score was used as a control parameter of these correlations (Table IV). As a result, the median Breslow score in the metastatic group was statistically significantly higher than in the group without metastases.

OPG is a potent pro-angiogenic factor, it regulates bone turnover and has additional roles in the immune and vascular systems (9). OPG plays an important role in tumor angiogenesis. Production of OPG is thought to be part of tumor cell survival strategy. A number of studies have been performed assessing the role of OPG in tumorigenesis (10). Lau with collaborators proved the messenger RNA (mRNA) expression of OPG by primary melanoma cells and also by metastatic melanoma cells (11). This study explains our finding of elevated OPG plasma levels in the melanoma group compared to healthy controls (Table II). If we focus on the first Breslow score stage we can see that the use of OPG for early diagnostics of melanoma will be limited. Statistically significant increase of OPG levels was observed starting from the second Breslow score stage (Table III). OPG plasma levels were increased with statistical significance in patients with sentinel lymph node metastases positivity (Table IV), which is a promissing outcome with regards to the potential prognostic value of OPG.

OPN is an important factor in bone remodeling (12). OPN has been also described in the context of diverse physiological roles such as chemotaxis, cell migration, cell adhesion, 
Table II. Biomarker levels in healthy and melanoma group.

\begin{tabular}{|c|c|c|c|c|c|c|c|}
\hline Analyte (units) & Status & Count $(\mathrm{N})$ & Mean & Median & Minimum & Maximum & $p$-Value Wilcoxon test \\
\hline \multirow[t]{2}{*}{ OPG (pg/ml) } & Healthy & 139 & 244.7 & 234.1 & 120.8 & 513.3 & \multirow[t]{2}{*}{$<0.0001$} \\
\hline & Melanoma & 183 & 406.5 & 355.2 & 141.9 & 1,183 & \\
\hline \multirow[t]{2}{*}{ OPN (pg/ml) } & Healthy & 139 & 10,363 & 8,535 & 578.8 & 35,141 & \multirow[t]{2}{*}{$<0.0001$} \\
\hline & Melanoma & 183 & 19,220 & 14,957 & 3,020 & 147,866 & \\
\hline \multirow[t]{2}{*}{$\mathrm{EGF}(\mathrm{pg} / \mathrm{ml})$} & Healthy & 139 & 33.19 & 22.00 & 3.20 & 324.0 & \multirow[t]{2}{*}{0.0379} \\
\hline & Melanoma & 183 & 41.69 & 29.00 & 3.28 & 352.0 & \\
\hline \multirow[t]{2}{*}{ VEGF (pg/ml) } & Healthy & 139 & 206.5 & 87.24 & 16.00 & 1,472 & \multirow[t]{2}{*}{0.5111} \\
\hline & Melanoma & 183 & 379.3 & 107.2 & 16.22 & 9,896 & \\
\hline
\end{tabular}

Table III. Biomarker levels in Breslow score stages.

\begin{tabular}{|c|c|c|c|c|c|c|c|}
\hline Analyte (units) & $\begin{array}{c}\text { Status } \\
\text { Breslow } \\
\text { score }(\mathrm{mm})\end{array}$ & Count $(\mathrm{N})$ & Mean & Median & Minimum & Maximum & $p$-Value Wilcoxon test \\
\hline \multirow[t]{5}{*}{ OPG (pg/ml) } & Healthy & 139 & 244.7 & 234.1 & 120.8 & 513.3 & - \\
\hline & $<1$ & 52 & 321.8 & 287.6 & 141.9 & 608.5 & 0.0239 \\
\hline & $1-2$ & 79 & 364.5 & 323.9 & 162.5 & 631.7 & $<0.0001$ \\
\hline & $2-4$ & 35 & 389.4 & 398.0 & 179.4 & 808.6 & 0.0004 \\
\hline & $>4$ & 17 & 521.3 & 500.7 & 341.5 & 832.7 & $<0.0001$ \\
\hline \multirow[t]{5}{*}{ OPN (pg/ml) } & Healthy & 139 & 10,363 & 8535 & 578.8 & 35,141 & - \\
\hline & $<1$ & 52 & 13,774 & 12801 & 2,022 & 35,198 & 0.0327 \\
\hline & $1-2$ & 79 & 17,134 & 15345 & 588.7 & 45,817 & 0.0016 \\
\hline & $2-4$ & 35 & 20,225 & 18613 & 5,493 & 94,563 & $<0.0001$ \\
\hline & $>4$ & 17 & 30,245 & 24138 & 11,342 & 62,476 & $<0.0001$ \\
\hline \multirow[t]{5}{*}{$\mathrm{EGF}(\mathrm{pg} / \mathrm{ml})$} & Healthy & 139 & 33.19 & 22.00 & 3.20 & 324.0 & - \\
\hline & $<1$ & 50 & 21.64 & 21.00 & 5.00 & 37.00 & 0.7482 \\
\hline & $1-2$ & 79 & 42.26 & 32.00 & 3.00 & 166.0 & 0.0273 \\
\hline & $2-4$ & 35 & 69.31 & 32.00 & 3.00 & 352.0 & 0.0250 \\
\hline & $>4$ & 17 & 62.36 & 37.00 & 8.00 & 280.0 & 0.0287 \\
\hline \multirow[t]{5}{*}{ VEGF (pg/ml) } & Healthy & 139 & 206.5 & 87.24 & 16.00 & 1,472 & - \\
\hline & $<1$ & 50 & 120.1 & 80.56 & 16.00 & 369.4 & 0.5977 \\
\hline & $1-2$ & 79 & 456.0 & 131.6 & 16.00 & 2043 & 0.1016 \\
\hline & $2-4$ & 35 & 1,004 & 96.28 & 16.00 & 5249 & 0.4329 \\
\hline & $>4$ & 14 & 738.5 & 183.9 & 16.00 & 9868 & 0.1248 \\
\hline
\end{tabular}

Table IV. Biomarker levels and sentinel nodes.

\begin{tabular}{|c|c|c|c|c|c|c|c|}
\hline Analyte (units) & $\begin{array}{c}\text { Sentinel } \\
\text { node status }\end{array}$ & Count $(\mathrm{N})$ & Mean & Median & Minimum & Maximum & $p$-Value Wilcoxon test \\
\hline \multirow[t]{2}{*}{ OPG (pg/ml) } & Negative & 48 & 324.6 & 298.4 & 141.9 & 631.7 & \multirow[t]{2}{*}{0.2158} \\
\hline & Positive & 27 & 274.5 & 275.7 & 162.5 & 429.9 & \\
\hline \multirow[t]{2}{*}{ OPN (pg/ml) } & Negative & 48 & 19,634 & 14,247 & 2,553 & 45,364 & \multirow[t]{2}{*}{0.0021} \\
\hline & Positive & 27 & 28,586 & 25,244 & 15,986 & 94,563 & \\
\hline \multirow[t]{2}{*}{ EGF (pg/ml) } & Negative & 48 & 28.09 & 26.25 & 3.28 & 40.47 & \multirow[t]{2}{*}{0.0114} \\
\hline & Positive & 27 & 114.3 & 80.15 & 9.54 & 352.0 & \\
\hline \multirow[t]{2}{*}{ VEGF (pg/ml) } & Negative & 42 & 392.5 & 105.8 & 16.00 & 5,249 & \multirow[t]{2}{*}{0.0027} \\
\hline & Positive & 23 & 1,669 & 979.8 & 32.28 & 9,868 & \\
\hline \multirow[t]{2}{*}{ Breslow score (mm) } & Negative & 48 & 1.7 & 1.8 & 0.2 & 5.5 & \multirow[t]{2}{*}{0.0004} \\
\hline & Positive & 27 & 2.7 & 2.9 & 0.4 & 10.0 & \\
\hline
\end{tabular}


angiogenesis, apoptosis, cell-extracellular matrix interactions and tumour metastasis. OPN actively promotes the tumourigenic phenotype and contributes to metastasis (13).

High levels of OPN are associated with poor prognosis in a variety of cancers while overall and disease-free interval are inversely related to osteopontin levels $(14,15)$. Kadkol et al. and Barak et al. performed a study concerning metastatic uveal melanoma where plasma levels of OPN were significantly higher in patients with metastatic melanoma compared to patients with DFI for 10 years and levels of metastatic patients were also significantly higher than those without metastases $(16,17)$. Barak et al. also proved that OPN plasma levels increase during metastases formation and can be detected as early as 12-18 months prior to CT scans (18). Rangel et al. proved an association between high OPN expression and increased tumour thickness. OPN expression was also an independent significant predictor of sentinel lymph node metastases (19). Our results correlate with all the above mentioned findings. We found elevated OPN plasma levels in the melanoma group compared to healthy controls (Table II). The elevation of OPN was even observed at all individual Breslow score stages (Table III). OPN was also statistically significantly increased in patients with sentinel lymph node metastases positivity (Table IV). OPN levels positively correlated with the increasing tumour thickness. All these facts are promissing for OPN as a marker of early stages and also as a prognostic biomarker.

EGF stimulates growth, proliferation, and differentiation of the cells. According to several studies, EGF has been implicated as a factor indicating tumour progression in certain types of cancers (20). In the study conducted by Bracher et al., EGF was identified as an important factor in the mediation of melanoma lymph node metastases (21). Dadras, in their own study empphasized the crucial role of EGF in the generation of lymphatic vessels. Lymph node metastases are associated with these lymphatic vessels in human melanoma. Lymph node metastases are associated with poor prognosis among patients with melanoma (22). Our findings are in concordance with the findings in the above mentioned studies. EGF levels were found to be statistically significantly higher in the group of melanoma patients compared to the control group but mainly EGF levels were significantly higher statistically in the group of patients with sentinel lymph node metastases compared to the group without metastases (Table IV). This finding supports the ability of EGF to be a prognostic marker.

VEGF naturally occurs as a glycoprotein and is an important factor of vasculogenesis and angiogenesis. VEGF is overexpressed in almost all types of solid cancers (23). Boon et al. found no correlation between VEGF plasma levels and the Breslow score but there was a positive correlation with the sentinel lymph node metastases (24). These results were confirmed by Vihinen et al. or Lugowska et al. $(25,26)$. Tas et al. observed that circulating levels of VEGF were significantly elevated in patients with melanoma compared to healthy controls but didn't correlate with the Breslow score (27). Similar results were observed in a broad multimarker study from Yurkovetsky et al. (28). Ugurel et al. found that elevated plasma levels of VEGF strongly correlated with poor overall survival and disease-free interval (29). Pelletier et al. measured VEGF during follow-up and onbserved earlier relapse in the patients with the elevated levels of VEGF (30). Our results support the prognostic value of plasma VEGF levels due to statistically significant elevated levels in patients with sentinel lymph node metastases positivity compared to the patients with negative lymph node status (Table IV).

In conclusion, if we focus on the first Breslow score stage of melanoma we can conclude that plasma levels of OPG and OPN are promising biomarkers for use at early stages of melanoma. Besides, OPN can also be used as a prognostic marker. EGF and VEGF appear to be prognostic biomarkers in cases of cutaneous melanoma.

\section{Acknowledgements}

Supported by the Ministry of Health, Czech Republic - conceptual development of research organization (Faculty Hospital in Pilsen FNP1, 00669806).

\section{References}

1 Monshi B, Vujic M, Kivaranovic D, Sesti A, Oberaigner W, Vujic I, Ortiz-Urda S, Posch C, Feichtinger H, Hackl M and Rappersberger K: The burden of malignant melanoma--lessons to be learned from Austria. Eur J Cancer 56: 45-53, 2016.

2 Erickson C and Driscoll MS: Melanoma epidemic: facts and controversies. Clin Dermatol 28: 281-286, 2010.

3 National Cancer Institute, Surveillance Epidemiology, and End Results Program. European Cancer Observatory. [Accessed June 3, 2016].

4 Apalla Z, Lallas A, Sotiriou E, Lazaridou E and Ioannides D: Epidemiological trends in skin cancer. Dermatol Pract Concept 30: 1-6, 2017.

5 Gogas H, Eggermont AM, Hauschild A, Hersey P, Mohr P, Schadendorf D, Spatz A and Dummer R: Biomarkers in melanoma. Ann Oncol 20: 8-13, 2009.

6 Vereecken P, Cornelis F, Van Baren N, Vandersleyen V and Baurain JF: A synopsis of serum biomarkers in cutaneous melanoma patients. Dermatol Res Pract 2012: 260643, 2012.

7 Spatz A, Stock N, Batist G and van Kempen LC: The biology of melanoma prognostic factors. Discov Med 10: 87-93, 2010.

8 Wright BE, Scheri RP, Ye X, Faries MB, Turner RR, Essner R and Morton DL: The importance of sentinel lymph node biopsy in patients with thin melanoma. Arch Surg 143: 892-899, 2008.

9 Martinetti A, Bajetta E, Ferrari L, Zilembo N, Seregni E, Del Vecchio M, Longarini R, La Torre I, Toffolatti L, Paleari D and Bombardieri E: Osteoprotegerin and osteopontin serum values in postmenopausal advanced breast cancer patients treated with anastrazole. Endocr Relat Cancer 11: 771-779, 2004. 
10 Tsukamoto S, Ishikawa T, Iida S, Ishiguro M, Mogushi K, Mizushima H, Uetake H, Tanaka H and Sugihara K: Clinical significance of osteoprotegerin expression in human colorectal cancer. Clin Cancer Res 17: 2444-2450, 2011.

11 Lau YS, Sabokbar A, Giele H, Cerundolo V, Hofstetter W and Athanasou NA: Malignant melanoma and bone resorption. Br J Cancer 94: 1496-1503, 2006.

12 Anborgh PH, Caria LB, Chambers AF, Tuck AB, Stitt LW and Brackstone M: Role of plasma osteopontin as a biomarker in locally advanced breast cancer. Am J Transl Res 7: 723-732, 2015

13 Weber GF, Lett GS and Haubein NC: Osteopontin is a marker for cancer agressiveness and patient survival. Br J Cancer 103: 861-869, 2010.

14 Tsukamoto S, Ishikawa T, Iida S, Ishiguro M, Mogushi K, Mizushima H, Uetake H, Tanaka H and Sugihara K: Clinical significance of osteoprotegerin expression in human colorectal cancer. Clin Cancer Res 17: 2444-2450, 2011.

15 Shang S, Plymoth A, Ge S, Feng Z, Rosen HR, Sangrajrang S, Hainaut P, Marrero JA and Beretta L: Identification of osteopontin as a novel marker for early hepatocellular carcinoma. Hepatology 55: 483-490, 2012.

16 Kadkol SS, Lin AY, Barak V, Kalickman I, Leach L, Valyi-Nagy K, Majumdar D, Setty S, Maniotis AJ, Folberg R and Pe'er J: Osteopontin expression and serum levels in metastatic uveal melanoma - a pilot study. Invest Ophthalmol Vis Sci 47: 802806, 2006

17 Barak V, Frenkel S, Kalickman I, Maniotis AJ, Folberg R and Pe'er J: Serum markers to detect metastatic uveal melanoma. Anticancer Res 27: 1897-1900, 2007.

18 Barak V, Kaiserman I, Frenkel S, Hendler K, Kalickman I and Pe'er J: The dynamics of serum tumor markers in predicting metastatic uveal melanoma (part 1). Anticancer Res 31: 345-349, 2011.

19 Rangel J, Nosrati M, Torabian S, Shaikh L, Leong SP, Haqq C, Miller JR 3rd, Sagebiel RW and Kashani-Sabet M: Osteopontin as a molecular prognostic marker for melanoma. Cancer 112: 144-150, 2008.

20 Kucera R, Cerna M, Narsanska A, Svobodova S, Strakova M, Vrzalova J, Fuchsova R, Treskova I, Kydlicek T, Treska V, Pecen L, Topolcan $\mathrm{O}$ and Pazdiora P: Growth factors and breast tumors, comparison of selected growth factors with traditional tumor markers. Anticancer Res 31: 4653-4656, 2011.
21 Bracher A, Cardona AS, Tauber S, Fink AM, Steiner A, Pehamberger H, Niederleithner H, Petzelbauer P, Gröger M and Loewe R: Epidermal growth factor facilitates melanoma lymph node metastasis by influencing tumor lymphangiogenesis. J Invest Dermatol 133: 230-238, 2013.

22 Dadras SS: An unexpected role for EGF in lymphangiogenesismediated melanoma metastasis to sentinel lymph nodes. J Invest Dermatol 133: 14-16, 2013.

23 Ferrara N: Vascular endothelial growth factor as a target for anticancer therapy. Oncologist 9: 2-10, 2004.

24 Boone B, Blokx W, De Bacquer D, Lambert J, Ruiter D and Brochez L: The role of VEGF-C staining in predicitng regional metastasis in melanoma. Virchows Arch 453: 257-265, 2008.

25 Vihinen PP, Hilli J, Vuoristo MS, Syrjänen KJ, Kähäri VM and Pyrhönen SO: Serum VEGF-C is associated with metastatic site in patients with malignant melanoma. Acta 46: 678-684, 2007.

26 Ługowska I, Kowalska M, Zdzienicki M, Fuksiewicz M, Kamińska J, Szamotulska K and Rutkowski P: The prognostic role of clinical factors, VEGF, IL-8 and sTNF-R1 in cutaneous melanomas at locoregional stage: Pol Merkur Lekarski 32: 2227, 2012.

27 Tas F, Duranyildiz D, Oguz H, Camlica H, Yasasever V and Topuz E: Circulating levels of vascular endothelial growth factor(VEGF), matrix metalloproteinase-3(MMP-3) and BCL-2 in malignant melanoma. Med Oncol 25: 431-436, 2008.

28 Yurkovetsky ZR, Kirkwood JM, Edington HD, Marrangoni AM, Velikokhatnaya L, Winans MT, Gorelik E and Lokshin AE: Multiplex analysis of serum cytokines in melanoma patients treated with Interferon-alpha2b. Clin Cancer Res 13: 2422-2428, 2007.

29 Ugurel S, Rappl G, Tilgen W and Reinhold U: Increased serum concentration of angiogenic factors in malignant melanoma patients correlates with tumor progression and survival. J Clin Oncol 19: 577-583, 2001.

30 Pelletier F, Bermont L, Puzenat E, Blanc D, Cairey-Remonnay S, Mougin C, Laurent R, Humbert P and Aubin F: Circulating vascular endothelial growth factor in cutaneous malignant melanoma. Br J Dermatol 152: 685-689, 2005.

Received May 25, 2018

Revised June 8, 2018

Accepted June 11, 2018 